\title{
Seismic Retrofit of Masonry Buildings with Polymer Grid
}

\author{
Marco Mezzi ${ }^{1}$ and Alberto Dusi ${ }^{2}$ \\ 1. Department of Civil and Environmental Engineering, University of Perugia, via G. Duranti 93, Perugia 06125, Italy \\ 2. Numeria Consulting S.r.l, Galleria del Corso 3, Cremona26100, Italy
}

\begin{abstract}
The performance of masonry walls reinforced using innovative polymer grids embedded into thin plaster layers as a tool for the seismic enhancement of brick masonry buildings has been investigated by experimental tests. A number of diagonal compression, shear compression and out-of-plane tests were executed on sample panels; experimental activities included pseudo-dynamic and shaking table tests on infills and reduced scaled building mock-ups, respectively. The results of the experimental activities are presented and discussed. Experimental campaigns have been supported by theoretical and numerical investigations; based on the experimental data and on the results of detailed numerical simulations, simplified models for the design of the retrofitting intervention are hereinafter proposed. The models, calibrated on the experimental evidence, properly consider the collapse mechanisms as well as the grid effect in the evolution of the above mentioned mechanisms.
\end{abstract}

Key words: Masonry buildings, seismic enhancement, polymer grid, cost-effective, kinematic analysis.

\section{Introduction}

Despite the fact that masonry buildings are usually characterized by simple configurations and require very poor constructive technology, their seismic behavior show elements of complexity greater than those of new structural configurations associated with modern materials like steel and reinforced concrete. Masonry is indeed a composite material whose behavior depends on its mechanical characteristics: tensile and compressive strength, elastic and shear modulus. Given the fact that the tensile strength is almost null, cracks on surfaces subjected to tension can easily be induced, causing a subdivision of the panels into separate portions that can transform sections of the building in kinematisms, so that each rigid portion moves with respect to the other till the structure's collapse. Such mechanisms can develop in the plane of the panels as well as orthogonally to them, giving rise, respectively, to the so called "first mode" and "second

Corresponding author: Marco Mezzi, professor, research fields: structural design, earthquake engineering, advanced systems for seismic protection, base isolation, structural rehabilitation, restoration of historical construction, performance base seismic design. E-mail: m.mezzi@unipg.it. mode" collapse mechanisms [1]. Furthermore, the texture of the masonry, associated with the dimensions and the organization of the blocks, can induce other behaviors characterized by the loss of integrity of the structural elements that can collapse for the breaking of the internal links among the blocks.

The seismic behavior of a masonry building depends on three fundamental performance issues to be guaranteed:

- the preservation of the global integrity, with no separation into macro-elements, thus allowing for a box-like behavior with a redistribution of the horizontal forces among all the resisting elements;

- the capability of all the members to resist the forces induced by the seismic actions, i.e., the capability of sustaining the induced forces without reaching the ultimate displacement;

- the capability of the panels not to develop collapse mechanisms associated to the evolution of the kinematisms and the loss of integrity.

A number of techniques for the retrofit of unreinforced masonry buildings in seismic areas have been presented in literature in the last decades [2-4] and are available on the market. Amongst these, works providing for the reinforcement of the plaster layers 
with steel, glass fiber or carbon fiber grids are commonly used.

Polymer grids have recently emerged as a promising structural retrofit solution for historical and adobe constructions $[5,6]$, but they could also be used in the seismic upgrading of ordinary brick or stone masonry buildings [7], thus representing an alternative system able of overcoming some deficiencies of the other reinforcement systems (e.g., corrosion of the steel) while being effective in terms of costs-benefits ratio.

\section{Polymer Grid-Based System Description}

The retrofitting system presented in this paper consists in the insertion of a polymer grid embedded into a thin lime based mortar plaster. The grid is the "RichterGard RG TX" one, a stiff monolithic polymer grid with integral junctions, characterized by an isometric geometry resulting in apertures of equilateral triangles. Fig. 1 shows the grid mesh, the installation at a suitable distance from the masonry, the plastering.

The basic underlying idea in the use of polymeric grid is to improve the performance of the masonry by increasing its strength and its ductility.

\section{Experimental Activities}

A pre-requisite for the use of the proposed retrofitting technique is the assessment of the effectiveness of plaster reinforcement as strengthening tool.

\subsection{Tests on Masonry Panels}

In the initial phase of the research activities, a series of tests, mainly aimed at evaluating the actual influence of the grid in the mechanical behavior of the reinforced element, with particular attention to its response to the horizontal actions, were carried out on masonry panels made using bricks and "weak" mortar mixes. Tests, executed on bare, plastered, unreinforced and reinforced specimens, consisted in:

- diagonal compression tests on 18 brick masonry squared panels;

- shear compression tests on 12 brick masonry rectangular panels;

- out-of-plane (stability) tests on 12 brick masonry large panels;

- out-of-plane (stability) tests on 4, previously damaged, repaired panels;

- shear and flexural tests on complex 3D elements of connections.

A detailed description of the aforesaid tests is given in [8]; for sake of conciseness, in the following (Figs. 2 and 3), selected pictures of the experimental set-up are shown along with some comments on the main outcomes.

Diagonal compression tests, carried out according to the ASTM standard [9], showed that the ultimate deformation of reinforced panels was increased by a factor of 2 to 3, which indicated that the grid's presence adds a positive contribution the global ductility.

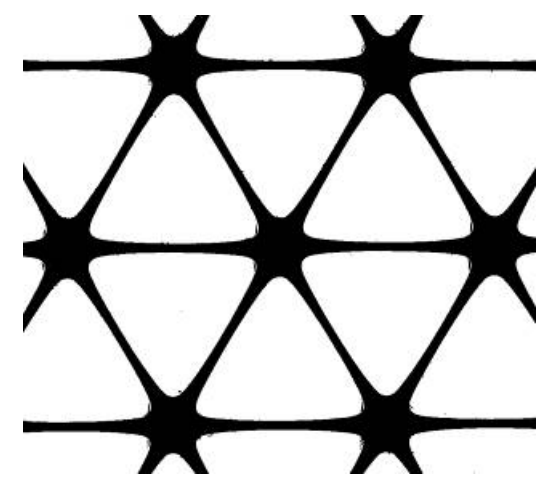

(a) grid

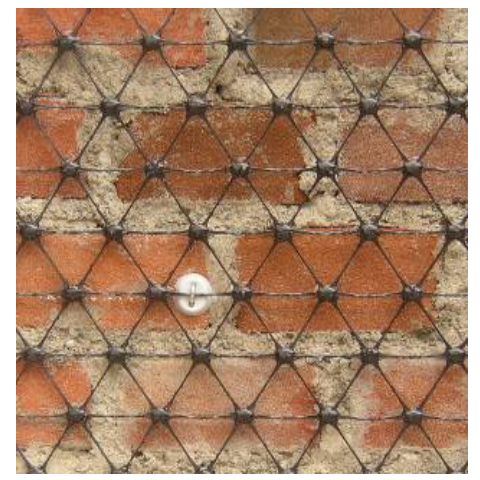

(b) installation of the grid

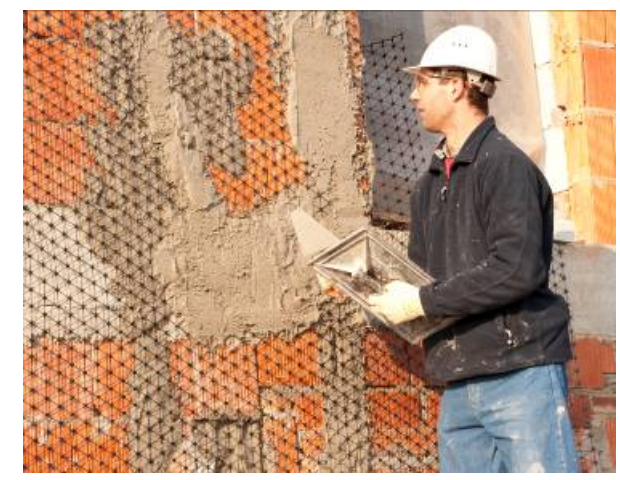

(c) plastering

Fig. 1 Characteristics of grid and its installation. 


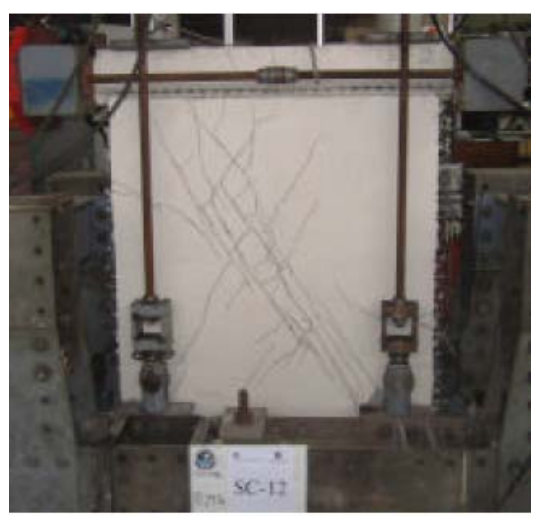

(a) Experimental setup

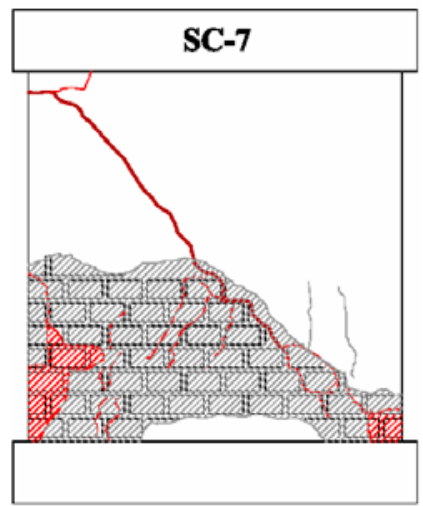

(b) Crack pattern in unreinforced panel

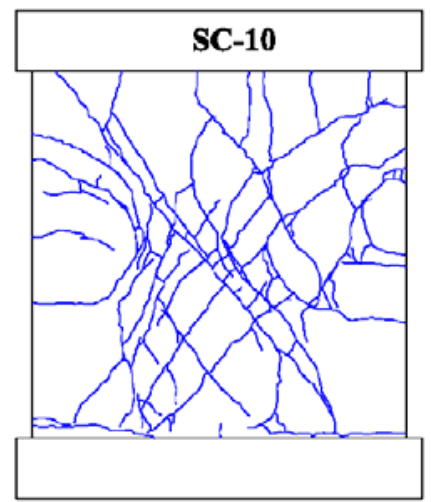

(c) Crack pattern in reinforced panel

Fig. 2 Shear-compression test.

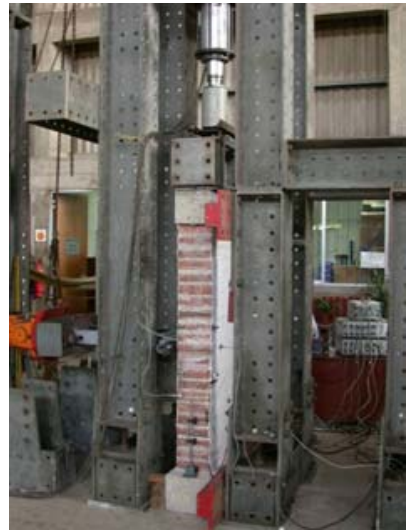

(a) Experimental set-up

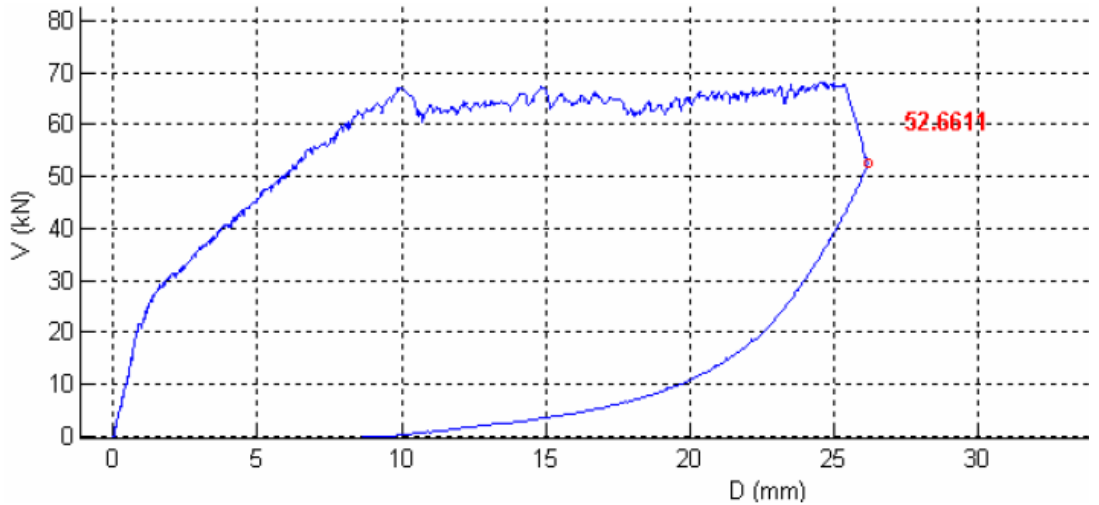

(b) Force-displacement curve of the reinforced panel

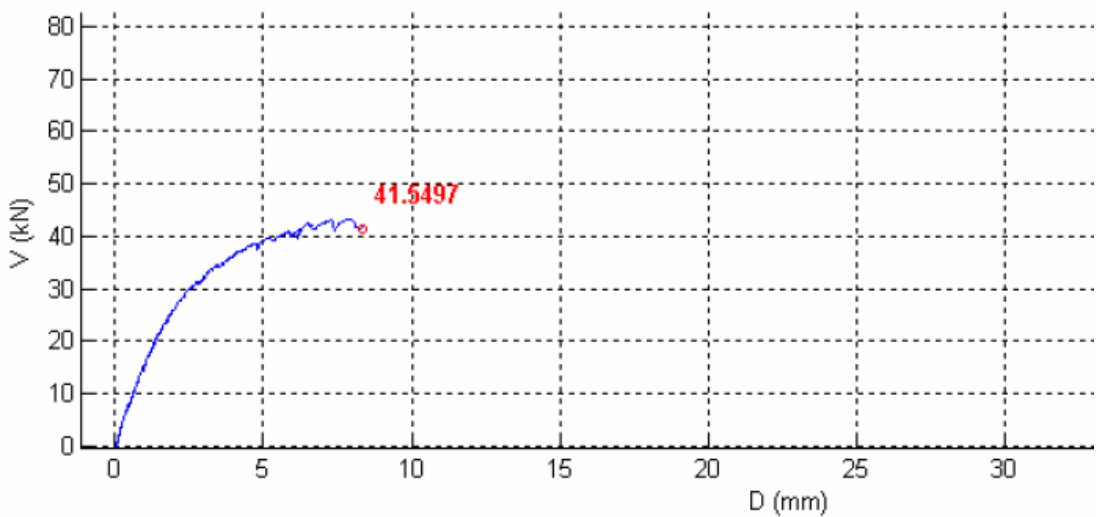

(c) Force-displacement curve of the bare panel

Fig. 3 Flexural test.

The results from shear-compression tests basically confirmed those from the diagonal-compression tests. The comparative observation of the unreinforced and reinforced panels' conditions at failure, though presenting similar modalities, showed very "clean" cracks diagonally orientated in the unreinforced panel, whilst the reinforced panels were characterized by widespread nets of cracks; this suggests that the reinforced panels' collapse requires the formation of a large number of failure surfaces and, therefore, an higher value of the ultimate strength. The shear-compression tests also confirmed the positive 
effect of the grid on the ductility of the panels as well as a significant energy dissipation capacity given by the grid. A non-negligible increment of the maximum resistant shear force was found.

The stability tests clearly demonstrated the positive effects of the grid reinforcement on all the significant mechanical parameters of the panels, i.e., ultimate load, ultimate displacement and energy dissipation. The distribution of the crack patterns, already observed in shear compression tests, put again into evidence the beneficial contribution of the grid, related to the mitigation of the damage peak and to the increase in energy dissipation due to the spreading of the damaged areas. The analysis of the experimental data also confirmed the expected beneficial effects of the grid on the out-of-plane behavior of the panel, proven by a significant enhancement of the resistance against the collapse mechanisms.

\subsection{Pseudo dynamic and Shake Table Tests}

A second phase of research focused on pseudo dynamic tests (reaction wall) on 2D full scale infill and on shake table tests on 3D scaled brick and stone masonry building mock-ups. In order to evaluate the effect of the grid shapes, a biaxial polymer grid was used in these tests. Reaction wall test and shake table tests are extensively reported in [5-10] respectively. Fig. 4 shows the response of the plain and of the reinforced panels.

The results of the tests demonstrate the effectiveness of the adoption of the polymer grid in increasing the energy dissipation capacity of masonry structures. The comparison between the behavior of the tested

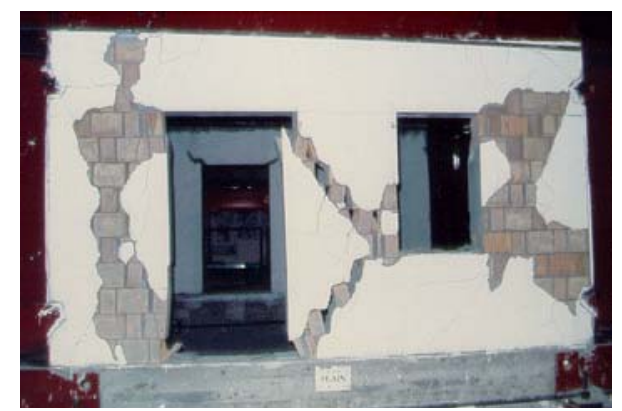

(a) Final state of the plain panel

Fig. 4 Reaction wall tests. mock-ups showed that the adoption of this methodology could strongly modify the strength-decay characteristics of the elements and that the proposed provisions also result in a shift of the ultimate shear strength towards larger forces and deformations.

\section{Evaluation of the Global Seismic Enhancement}

The effects of the plaster reinforcement consist in an increase of both strength and ductility.

\subsection{Strength Increase}

Different results on the strength increment have been obtained from the different tests carried out. Diagonal compression tests did not show significant shear strength increase of the panels; experimental results reported in literature confirm this behavior [11]. On the contrary, from shear-compression tests some non negligible increment of the maximum resistant shear force were found, but with peak effects. The discrepancy can be explained by considering that, besides the different testing modalities, diagonal-compression tests were carried out on masonry panels built with high strength bricks, while in the shear compression tests, use of medium strength bricks was made. Taking into account that existing buildings are often made of poor quality masonry, it can be stated that the polymeric grid reinforced plaster gives a shear strength increase. Such a strength increment can therefore be assumed in the design, with values ranging from 1.0 (no increase) up to 1.5 (maximum experimental finding); a 1.2 value is prudentially suggested by the authors.

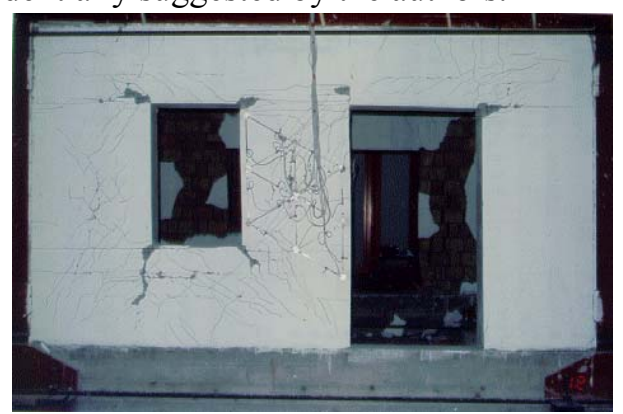

(b) final state of the reinforced panel 


\subsection{Ductility Increase}

The increment in the ductility capacity, given by the presence of polymeric grid, is a constant result of all the tests carried out, therefore the fact that the reinforcement increases significantly the ductility is proven and must be considered in the design of the retrofit interventions. This effect can be directly taken into account when nonlinear static or dynamic analyses are carried out, for which the force-deformation relationship of each structural element is explicitly defined. According to the experimental results, the force-deformation diagrams of the reinforced panels can be characterized by an ultimate deformation of $0.4 \%$ (shear distortion). On the contrary, the maximum distortion of the tested unreinforced panels turned out to be approximately $0.2 \%$. It can be observed that the ultimate displacements obtained from the tests carried out, are lower than those found in literature for either new or ancient masonry $[12,13]$. The more pronounced plastic behavior shown in the literature cases could be attributed to the mortar characteristics and to the masonry organization: this aspect will be clarified by further tests, already planned, on panels made with low quality masonry.

\subsection{Modality for Seismic Check}

The increase in ductility of the reinforced masonry, as well as the strength increase, can be taken into account within an analysis method for the seismic assessment of the building, through the amplification of the structure factor $q$. Considering, for an existing building, a strength increase $k_{s}$ (i.e., 1.2) and a ductility increase $k_{\mu}$ (i.e., 2.0), an amplification $k_{q}=k_{s} \times k_{\mu}$ could be assumed by the designer for the structure factor. If, more realistically, an energy-based correlation between ductility and structure factor is assumed, the amplification factor is given by the following expression:

$$
k_{q}=k_{s}+\sqrt{\frac{k_{\mu}\left(q_{0}^{2}+1\right)-1}{q_{0}^{2}}}
$$

where $q_{0}$ is the basic structure factor.

\section{Assessment Based on Local Collapse Mechanisms}

The experimental activities have been supported by theoretical and numerical investigations aimed at interpreting, reproducing the results and defining suitable simplified models to be used as tools for the design of the retrofitting interventions [14].

As previously said, in the existing masonry buildings partial collapses for seismic actions often happen, generally for loss of equilibrium of masonry portions. The verification with reference to the in-plane and out-of-plane collapse mechanisms, is meaningful only if a monolithic behavior of the masonry wall is guaranteed. Under this assumption, the verification can be carried out by resorting to the limit analysis of the equilibrium, according to the kinematic approach, based on the identification of a collapse mechanism and on the evaluation of the horizontal force that activate such a mechanism. The application of the proposed verification method presupposes, therefore, the analysis of the local mechanisms, deemed significant for the construction. These latter can be assumed from the knowledge of the seismic behavior of similar structures, already damaged from the earthquake, or can be defined by considering the presence of crack patterns, even if not directly related to seismic actions. The quality of the connections among the walls, the masonry organization and texture, the presence of tie-bars, the interactions with other elements of the construction or the neighboring buildings must also be considered.

The kinematic approach also allows for the determination of the horizontal force evolution that the structure is progressively able to withstand meanwhile the mechanism evolves. Having defined $\alpha$ as the ratio of the horizontal forces applied to the correspondents weights of the structural masses, such an evolution can be represented by a curve of $\alpha$ multiplier as a function of the displacement $d_{k}$ of a reference point in the 
system. The curve, determined up to the annulment of any capability of sustaining the horizontal actions $(\alpha=$ 0 ), can be transformed into a capacity curve of an equivalent single-degree-of-freedom system, for which the ultimate displacement capacity of the local mechanism can be defined and compared to the displacement demand requested by the seismic action.

For the application of the analysis method, the following assumptions are generally made:

- the masonry tensile strength is null;

- absence of sliding among the blocks;

- the masonry compressive strength is infinite.

However, for a more realistic simulation, the following parameters shall be considered, even if in a simplified way:

- the sliding between the blocks, by taking into account the friction;

- the connections (even if of limited resistance) among the walls;

- the presence of metallic tie-rods;

- the presence of the polymeric grid within the plaster and on the corners;

- the limited compressive strength of the masonry, by considering sets of hinges adequately located;

- the presence of multi-leaf walls.

\subsection{Linear Kinematic Analysis}

In order to get an evaluation of the horizontal loads multiplier $\alpha_{0}$ that leads to the activation of the local mechanism, it is necessary to apply the following forces to the rigid blocks composing the kinematic chain:

- the self weights of the blocks (applied to their centre's of mass);

- the dead and live vertical loads acting on the blocks;

- a system of horizontal forces proportional to vertical loads (if not transmitted to others walls);

- other external forces (e.g., those transmitted by metallic tie-rods);

- other internal forces (e.g., actions related to the block interconnections and the grid presence).
Assigning a generalized virtual displacement to the generic block $k$ of the kinematic chain, one can determine, as a function of the rotation and geometry of the structure, the displacements components of application points of the various forces applied in their respective directions. The multiplier $\alpha_{0}$ is then obtained by applying the Principle of the Virtual Work and equating the work done by the internal and external forces applied to the system acting through the virtual displacements:

$\alpha_{0}\left(\sum_{i=1}^{n} P_{i} \delta_{x, i}+\sum_{j=n+1}^{m} P_{j} \delta_{x, j}\right)-\sum_{i=1}^{n} P_{i} \delta_{y, i}-\sum_{h=1}^{o} F_{h} \delta_{h}=L_{f i}$

where:

$n$ is the number of all the forces applied to the various blocks of the kinematic chain;

$m$ is the number of forces not directly acting on the blocks, generating horizontal forces on the elements of the kinematic chain;

$o$ is the number of external forces, not associated to masses, applied to the various blocks;

$P_{i}$ is the vertical force of the generic block;

$P_{j}$ is the generic vertical force, not directly applied to the blocks;

$\delta_{x, i}, \delta_{x, j}$ are the horizontal virtual displacements of the points of application of the forces $P_{i}$ and $P_{j}$;

$\delta_{y, i}$ is the vertical virtual displacement of the point of application of load $P_{i}$;

$F_{h}, \delta_{h}$ are, respectively, the external force applied to a block and the displacement of the application point;

$L_{f i}$ represents the work of the internal forces.

\subsection{Nonlinear Kinematic Analysis}

In order to evaluate the displacement capacity of the structure, up to its collapse, in the considered mechanism, the horizontal load multiplier $\alpha_{0}$ can be determined not only with reference to the initial configuration, but also to modified configurations of the kinematic chain, representatives of the evolution of the mechanism and defined by the displacement $d_{k}$ of a system's reference point. The analysis must be carried 
out up to the configuration corresponding to the annulment of the $\alpha$ multiplier, corresponding to a displacement $d_{k, 0}$. For any configuration of the kinematism, the value of $\alpha_{0}$ can be determined from equation 2, properly rewritten by referring to the modified geometry. The analysis can be carried out with the use of graphic methods, by defining the system geometry in the different configurations up to its collapse, or with analytical-numerical methods, by considering a set of virtual displacements and rotations to be progressively updated based on the system geometry evolution.

If the forces involved are kept constant during the kinematism evolution, the resulting curve is almost linear. When the progressive variation of the external forces with the kinematism evolution is taken into account (e.g., when considering the elongation of the grid within the plaster), the curve could be linearized by segments, evaluating the curve in correspondence to displacements for which significant variations happen (i.e., grid yielding, ultimate deformation of the grid).

\section{Out of Plane Collapse Mechanisms}

In the following is reported, for its significance, an example of application of the proposed analysis approach to out-of-plane collapse mechanism, validated on the basis of the experimental stability test results. The analysis is based on the assumption that collapse mechanism consists of a horizontal crack located at an unknown level. The crack divides the masonry panel into two "rigid" portions (block 1 and block 2) as shown in Fig. 5.

Given the virtual rotation $\theta_{l}$ of block 1 , the rotation $\theta_{2}$ of block 2 can be easily computed. The equilibrium condition can therefore be derived from the Principle of Virtual Works by equating the total work done by the external and internal forces that are applied to the considered system along the correspondent virtual act of motion. The forces doing the work along the system's virtual displacement are:
- the weight of the lower portion $P_{1}=P \cdot a_{1} / h=P \cdot(x-1) / x$ and that of the upper portion $P_{2}=P \cdot a_{2} / h=P / x$;

- the lateral seismic forces related to the self weight, $F_{1}=\alpha_{0} \cdot P_{1}$ and $F_{2}=\alpha_{0} \cdot P_{2}$, given as a function of $\alpha_{0}$, the multiplier of the actions that activate the mechanism;

- the vertical reaction at the top of the wall, $V$ (including the loads originating from the overhanging walls and floors);

- the resistant moment of the cracked reinforced section $M_{\text {res }}$, evaluated considering the grid's presence. $M_{\text {res }}$ can be computed considering the ultimate limit state of the section with the grid in correspondence of the stretched edge. The calculation also allows for the definition of the neutral axis position, which identifies the axis around which the rotations $\theta_{2}$ develop.

The displacements of the forces' application points, i.e., the rotations of the moment application axes, can be easily derived from geometric considerations on the kinematism (Fig. 5).

From the application of the Principle of Virtual Works, it can be obtained:

$F_{1} \cdot \delta_{1 x}+F_{2} \cdot \delta_{2 x}-P_{1} \cdot \delta_{1 y}-P_{2} \cdot \delta_{2 y}-V \cdot \delta_{v y}-M_{r e s} \cdot x=0$

from which, by simple substitutions, the value of the $\alpha_{0}$ seismic multiplier of the actions activating the mechanism can be obtained.

From a general point of view, the value of $\alpha_{0}$ shall be compared with the seismic design coefficient, therefore the verification is satisfied when $\alpha_{0} \geq \lambda S_{d}$, being $S_{d}$ the value, in $g$, of the design spectrum, i.e. the value of the
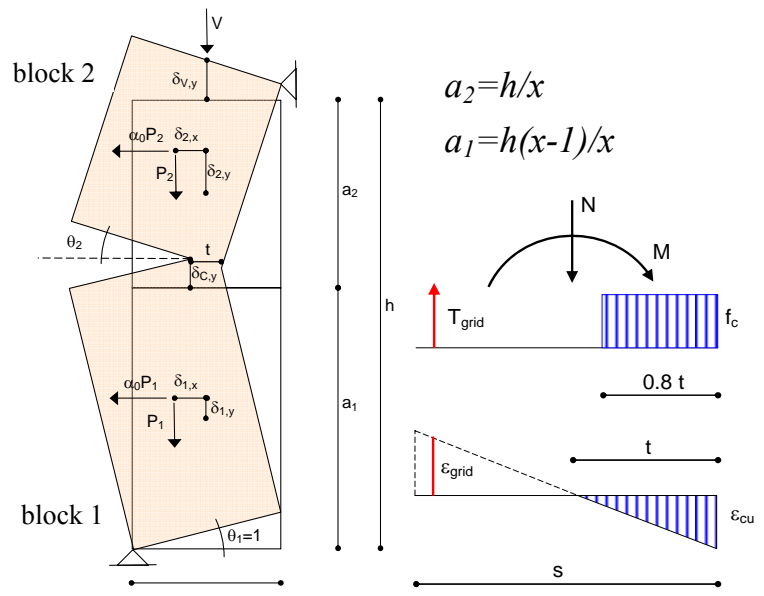

Fig. 5 Out-of-plane collapse of a panel. 
elastic spectrum divided by the proper $q$ factor, and $\lambda$ is the reduction factor of the seismic forces.

From the tests carried out applying loads orthogonal to the panel [14], a value of $q=3$ can be assumed. In this case, $\alpha_{0}$ is defined as a function of $x$, the elevation of the collapse section, that can be estimated by minimizing the value of the $\alpha_{0}$ multiplier, by imposing

$$
\frac{d}{d x} \alpha_{0}=0
$$

from which

$$
\alpha_{0}=\frac{s}{h\left(x_{0}-1\right)}\left[2 x_{0}+\frac{V \cdot x_{0}}{P}+\frac{V \cdot x_{0}^{2}}{P}-\frac{2 \cdot t \cdot x_{0}}{s}-\frac{2 V \cdot t \cdot x_{0}^{2}}{s \cdot P}+\frac{2 x_{0}^{2} \cdot M_{r e s}}{s \cdot P}\right]
$$

Because $x_{0} \geq 1$, the contribution of the term

$$
\frac{2 \cdot x_{0}^{2} \cdot M_{r e s}}{P \cdot h \cdot\left(x_{0}-1\right)}
$$

associated to the resisting moment of the cracked section, is always positive and therefore represents an increment of the load factor $\alpha_{0}$.

Similar approaches can be extended to nonlinear analysis and to overturning as well as in-plane collapse mechanism.

\section{Pilot Applications to Real Buildings}

Pilot applications of the polymer grid as plaster reinforcement have been already carried out on some simple historical buildings. The building hosting the Visitor Centre of the Natural Monument of Campo Soriano in Italy is a tuff masonry building included in the Italian cultural heritage (Fig. 6). In the framework of the works for the complete restoration and the seismic enhancement of the building, all the masonry panels have been plastered including a polymer grid (Fig. 7) of a type similar to the one illustrated in the present paper.

Another sample building is the Social Centre Ex GIL of the Municipality of Formia (Italy). It is a historical building made of stone masonry that was partially ruined as a consequence of a long state of abandon that determined a strong degradation of the construction (Fig. 8).

Fig. 9 reports, with reference to the damaged façade shown in Fig. 8, the design works foreseen in the refurbishment and seismic enhancement design. The design provided, among the other works, for the reconstruction of the lost portions of the building with

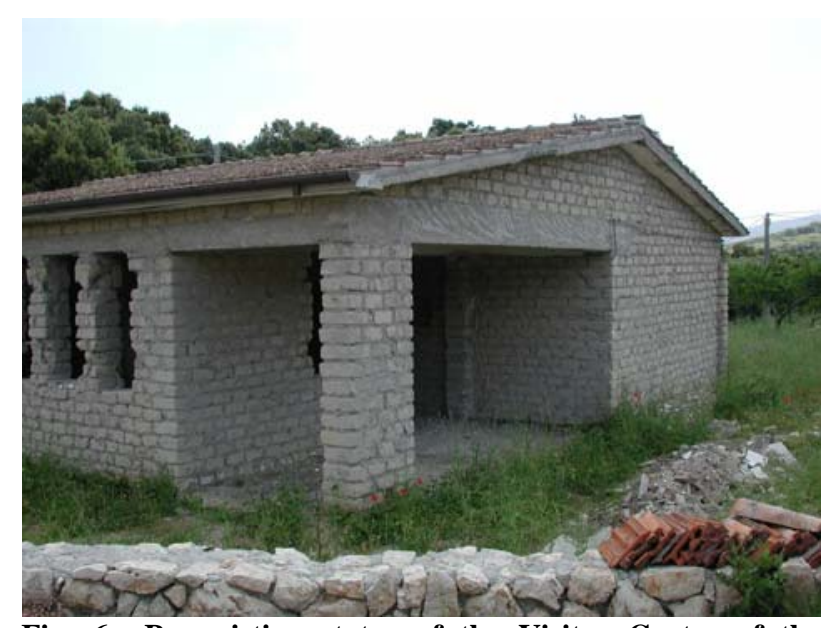

Fig. 6 Pre-existing status of the Visitor Centre of the Natural Monument of Campo Soriano (Italy).

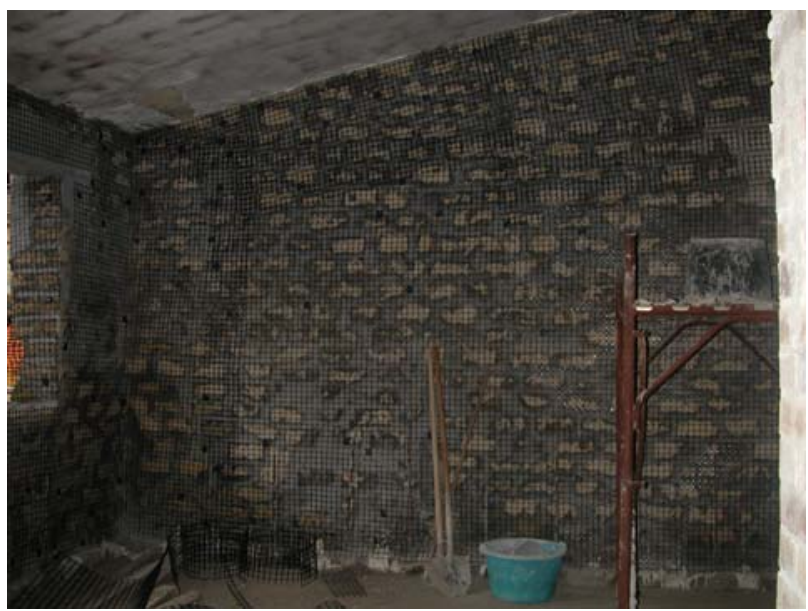

Fig. 7 Visitor Centre of Campo Soriano (Italy): polymer grid installed before plastering.

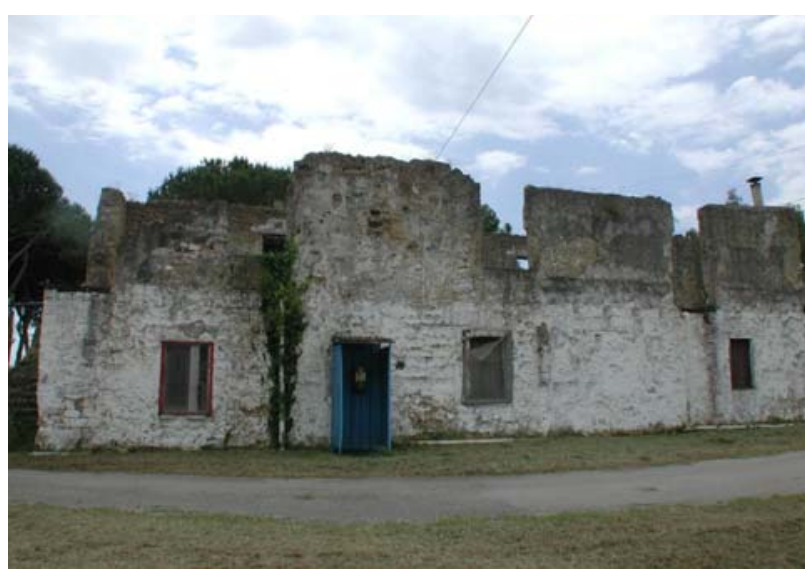

Fig. 8 Ruined status of the Social Centre Ex GIL in the Municipality of Formia (Italy). 


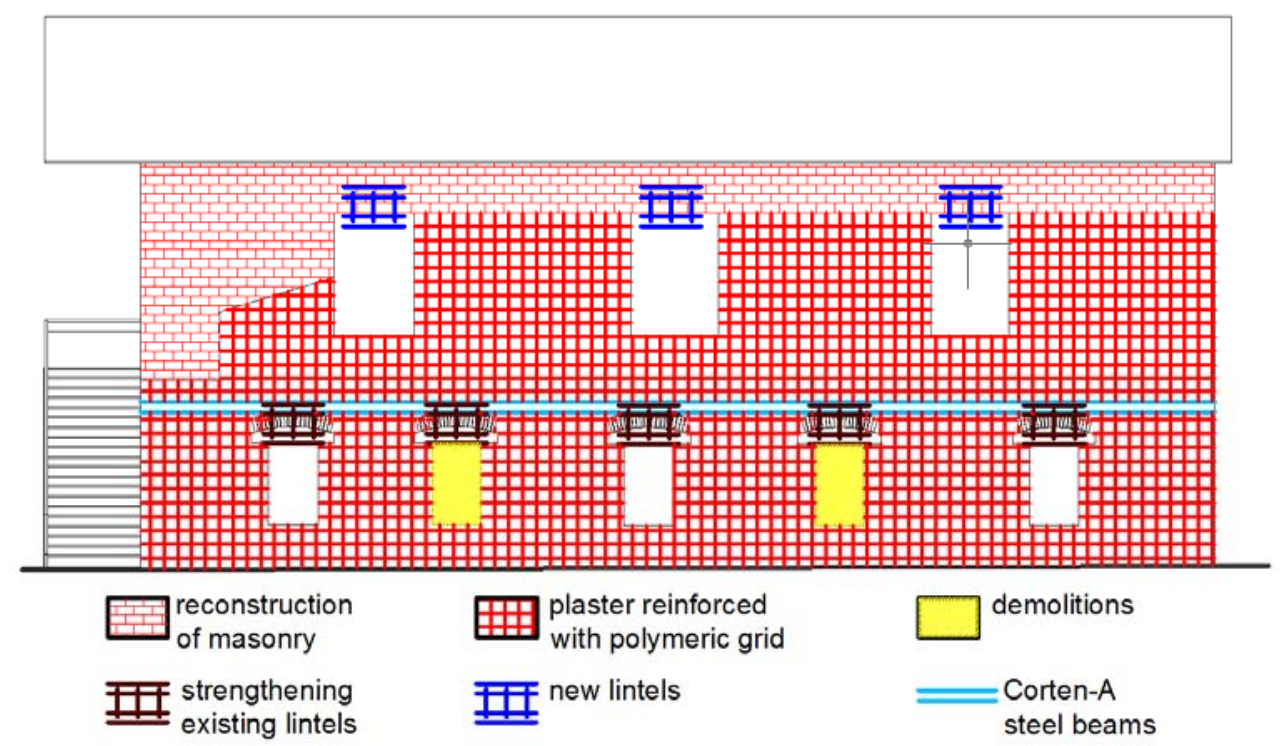

Fig. 9 Main works designed for the refurbishment and seismic enhancement of the Social Centre Ex GIL in the Municipality of Formia (Italy).

new brick masonry and the consolidation of the existing stone masonry by means of cement based mortar plastering reinforced with a polymer grid like the one illustrated in the present paper.

\section{Conclusions}

Experimental campaigns proved that plaster reinforced with polymer grid can be an effective, simple and a low-cost solution for the seismic performance enhancement of common masonry buildings as well as of historical buildings. Critical aspects related to the disagreement among the test results, the results reported in literature [10-12] and the code provisions [15] in the definition of the ultimate displacement of masonry panels are evidenced and discussed.

In all the tests, the collapse modalities are similar for all the variants of the panels indeed they basically depend on the structural scheme of the panel under test. The fundamental difference among the variants consists of the cracking pattern. The unplastered and unreinforced panels show localized cracks, whilst the reinforced panels show a large diffusion of the cracks.

The grid does not significantly increase the strength of the masonry, even if a certain influence results from the shear-compression tests. Tests show that the effectiveness of the grid in the enhancement of the masonry performances is related to the inhibition of breaking up and collapse, indeed after the first damage; the grid prevents the collapse in separate portions. The contribution is also relevant to the out-of-plane failures increasing the safety factor of the so called first mode mechanisms.

The most relevant contribution consists of the increase of the ultimate deformation, i.e., of the ductility associated to in-plane collapse mechanisms, due to the link effect among the portions.

Simplified analytical tools have been developed for evaluating the strength and ductility of the reinforced panels with reference to both the "first mode" and "second mode" mechanisms. The seismic analyses are based on the use of design spectra reduced through a suitably defined behavior spectrum. Provisions of the suitable values of the behavior factor taking into account the strength and ductility increase given by the plaster reinforcement are given, based on the experimental evidence.

\section{References}

[1] A. Giuffrè, Letture sulla meccanica delle murature storiche, Kappa, Roma, Italy, 1990. (in Italian) 
[2] M. ElGawady, P. Lestuzzi and M. Badoux, A review of conventional seismic retrofitting techniques for URM, in: The 13th International Brick and Block Masonry Conference, Amsterdam, Netherland, July 2004.

[3] S. Frumento, S. Giovinazzi, S. Lagomarsino and S. Podestà, Seismic retrofitting of unreinforced masonry buildings in Italy, in: NZSEE Conference, Napier, New Zealand, 2006.

[4] Y. Zhuge and S. W. Chuang, Seismic retrofitting of unreinforced masonry buildings - A literature review, Australian Journal of Structural Engineering 6 (1) (2005) 25-36.

[5] CASCADE Project, Application of reinforcing techniques with polymer grids for masonry buildings, in: Severn R. \& Bairrao R. (General Editors), Cooperative Advancements in Seismic and Dynamic Experiments, Report No. 5, 2005.

[6] D. Torrealva, C. Cerrón and Y. Espinoza, Shear and out of plane bending strength of adobe walls externally reinforced with polypropylene grids, in: The 14th World Conference on Earthquake Engineering, Beijing, China, Oct. 2008.

[7] E. Manzoni, A. Dusi and M. Mezzi, Polymeric grid for a cost effective enhancement of the seismic performance of masonry buildings, in: The 14th World Conference on Earthquake Engineering, Beijing, China, Oct. 2008.

[8] M. Mezzi, A. Dusi and E. Manzoni, Polymeric grid for the rehabilitation of masonry structures, in: The 10th International Conference on Inspection, Appraisal, Repairs and Maintenance of Structures, Hong Kong, Oct. 2006.
[9] American Society for Testing Materials E 519-81, Standard Test Method for Diagonal Tension (Shear) in Masonry Assemblages, 1981.

[10] A. Colombo, P. Negro and G. Verzelletti, Improving ductility and energy-dissipation capacity of infills by means of polymeric nets, in: The 12th World Conference on Earthquake Engineering, 2000, Auckland, New Zealand.

[11] M. Drdácký and J. Lesák, Tests of brick masonry walls strengthened with polymer grids and subjected to combination of compression and cyclic shear, Research report ÚTAM AV ČR - CD, ÚTAM Praha, Czech Republic, 2001.

[12] A. Anthoine, G. Magenes and G. Magonette, Shear-compression testing and analysis of brick masonry walls, in: The 10th European Conference on Earthquake Engineering, 1994, Vienna, Balkema, Rotterdam, The Netherlands, 1995.

[13] S. Chiostrini, L. Galano and A. Vignoli, On the determination of strength of ancient masonry walls via experimental tests, in: The 12th World Conference on Earthquake Engineering, Auckland, New Zealand, 2000.

[14] A. Dusi, E. Manzoni and M. Mezzi, L'impiego di reti polimeriche per la riabilitazione di strutture in muratura - 2: Analisi, in: ANIDIS 2007 XII Convegno L'Ingegneria Sismica in Italia, Pisa, Italy, 2007. (in Italian)

[15] NTC 2008, Norme tecniche per le costruzioni D.M. Ministero Infrastrutture e Trasporti 14 gennaio 2008, G.U.R.I., Roma, Italy, Feb. 4, 2008. (in Italian) 